\title{
Temperature Compensated Solution Concentration Measurement Based on a Cascaded SMS/LPFG Fiber Structure
}

\author{
Jin Wang, Li Wang*, Xueqiong Su, Huanhuan Yan \\ College of Physics and Optoelectronics, Faculty of Science, Beijing University of Technology, Beijing, China \\ Email: *Lwang.1@bjut.edu.cn
}

How to cite this paper: Wang, J., Wang, L., Su, X.Q. and Yan, H.H. (2021) Temperature Compensated Solution Concentration Measurement Based on a Cascaded SMS/LPFG Fiber Structure. Optics and Photonics Journal, $11,294-300$

https://doi.org/10.4236/opj.2021.118019

Received: April 1, 2021

Accepted: July 31, 2021

Published: August 3, 2021

\begin{abstract}
In this paper, a hybrid optical fiber structure for solution concentration measurement with the temperature compensation is proposed. The structure consists of long period fiber grating (LPFG) and single mode-multimode-single mode (SMS) fiber structures. The sensing mechanism of the device is studied and verified by experiments. LPFG is sensitive to solution concentration and is affected by temperature crosstalk. SMS structure is not affected by solution concentration, but sensitive to ambient temperature. It can be used as a temperature compensation system. The sensitivity coefficients of LPFG and SMS on temperature and concentration were measured experimentally, and a dual-wavelength matrix was established to realize simultaneous measurement of solution temperature and concentration.
\end{abstract}

\section{Keywords}

Long Period Fiber Grating, Multimode Mode Fiber, Solution Concentration, Temperature, Dual-Wavelength Matrix

\section{Introduction}

Corrosion is the destruction or deterioration of materials or their properties in the environment. Salt spray corrosion is one of the most common and destructive forms of corrosion. Salt fog here refers to chloride, its main component is chloride salt-sodium chloride ( $\mathrm{NaCl})$ [1] [2] [3]. Therefore, it is very important to measure the concentration of $\mathrm{NaCl}$ to monitor the corrosion intensity, so as to avoid the impact of corrosion in engineering. In practical engineering, it is inconvenient and lack of real-time to collect samples manually to measure the concentration of $\mathrm{NaCl}$ in salt spray or solution. LPFG has the advantages of be- 
ing sensitive to the surrounding refractive-index, low insertion loss and working in high corrosive and dangerous environment. In addition, LPFG can be easily connected with computer and optical fiber transmission system for real-time measurement [4] [5]. Monitoring the liquid concentration by LPFG can not only save the labor of manual sampling, but also detect the concentration accurately in real time. Accurate measurement and on-site monitoring of liquid concentration are very important in chemical and biological fields. However, in the process of measurement, liquid concentration measurements are unreliable because temperature changes will interfere with the sensor. The influence of temperature change on the accuracy of concentration measurement is eliminated. In this paper, we propose a simple and feasible hybrid fiber structure, LPFG and SMS fiber cascade. LPFG will be affected by change of temperature and solution concentration, while SMS structure is only affected by temperature and is insensitive to the solution concentration. It can be used as a temperature compensator to eliminate the influence of temperature changes when measuring concentration.

\section{Experimental Principle}

The LPFG couples the core mode $\left(\mathrm{LP}_{01}\right)$ to the cladding modes of the same azimuthally symmetry $\left(\mathrm{LP}_{0 \mathrm{~m}}\right)$. Here, the resonance wavelength $\lambda_{D}^{m}$ satisfying the phase-matching condition is given by,

$$
\lambda_{D}^{m}=\left(n_{e f f}^{c o}-n_{e f f}^{c l, m}\right) \cdot \Lambda
$$

where $\lambda_{D}^{m}$ is the resonance wavelength, $\Lambda$ is the periodicity of the grating, $n_{\text {eff }}^{c o}$ and $n_{e f f}^{c l, m}$ are the effective refractive indices in the core and cladding respectively. $n_{\text {eff }}^{c o}$ is determined by core radius $a_{1}$, core refractive index $n_{1}$ and cladding refractive index $n_{2}, n_{\text {eff }}^{c l, m}$ is determined by core and cladding radius $a_{1}, a_{2}$ and refractive index of core, cladding and external environment $n_{1}, n_{2}, n_{3}$. The parameters $a_{1}, a_{2}, n_{1}, n_{2}, n_{3}$ remain unchanged, and $\lambda$ is determined only by the refractive index of the external environment. When the refractive index of the solution to be measured changes and $n_{3}$ becomes $n_{3}^{\prime}$, the expression of the relationship between the central wavelength shift of LPFG and the refractive index of the external environment is obtained as follows Formula (2). There is a linear relationship between the concentration and the refractive index of solution [6] [7] [8].

$$
\Delta \lambda=\lambda^{\prime}-\lambda_{0}=\frac{U_{\infty}^{2}}{n_{2} a_{2}^{3} K^{3}}\left(\frac{1}{\sqrt{n_{2}^{2}-n_{3}^{2}}}-\frac{1}{\sqrt{n_{2}^{2}-n_{3}^{\prime 2}}}\right)
$$

Since the effective refractive index of the fundamental mode and cladding modes, and grating period are all functions of temperature, the two sides of the Formula (1) are divided into temperature $(T)$ differential and $\lambda_{D}^{m}$ is replaced by $\lambda_{\text {res }}^{m}$. The temperature sensitivity of LPFG is [9] [10],

$$
\frac{d \lambda_{\text {res }}^{m}}{d T}=\left(\frac{d n_{\text {eff }}^{c o}}{d T}-\frac{d n_{e f f}^{c l, m}}{d T}\right) \Lambda+\left(n_{\text {eff }}^{c o}-n_{e f f}^{c l, m}\right) \frac{d \Lambda}{d T}
$$


In the SMS structure, the fundamental mode transmitted in single mode fibers is coupled to multimode fibers, which excites the fundamental modes and higher modes in multimode fibers. Different modes interfere in the multimode core which results in the energy redistribution. The final interference optical spectrum is determined by multiple mode interferences [11]. When the temperature changes, the multimode optical fibers are affected by thermal optic effect and thermal expansion effect. The relationship between the coupling wavelength of SMS structure and temperature is expressed as follows [12],

$$
\frac{\Delta \lambda_{T, S M S}}{\lambda_{0}}=\frac{\partial n_{c o}}{n_{c o}} \frac{\Delta T}{\partial T}+2 \frac{\partial a}{a \partial T} \Delta T-\frac{\partial L}{L \partial T}=\left(a_{M M F}+\xi\right) \cdot \Delta T
$$

where $\lambda_{0}$ is the central wavelength, $n_{c o}$ is the effective refractive index of multimode fiber, $a$ is the core radius of multimode fiber, $L$ is the length of multimode fiber, $\alpha$ and $\xi$ are the thermal expansion coefficient and thermal-optical coefficient of multimode fiber, respectively.

Temperature and concentration coefficients of SMS and LPFG were measured respectively and substituted into Formula (5) to form a dual-wavelength matrix. The unique variation of temperature and concentration in solution environment was determined according to the simultaneous variation of two wavelengths.

$$
\left[\begin{array}{c}
\Delta \lambda_{L P F G} \\
\Delta \lambda_{S M S}
\end{array}\right]=\left[\begin{array}{cc}
K_{T_{L P F G}} & K_{R I_{L P F G}} \\
K_{T_{S M S}} & K_{R I_{S M S}}
\end{array}\right]\left[\begin{array}{c}
\Delta T \\
\Delta R I
\end{array}\right]
$$

\section{Experimental Process and Measurement Results}

In the measurement of LPFG Temperature and Solution Concentration, as shown in Figure 1, SLED broadband source (BBS) is used as the input. The waveform is smooth with the spectral width of $600-1700 \mathrm{~nm}$, which meets the need of grating for light source modulation. Optical spectrum analyzer (OSA, $\mathrm{AQ6317B}$ ) is used to receive the signal. The spectrum receiving range is 800 $1700 \mathrm{~nm}$, and the highest resolution is $0.01 \mathrm{~nm}$. It can fully display the spectral characteristics of grating modulation. The temperature change of grating is realized by temperature control box.

In the experiment of solution concentration measurement, LPFG is fixed at both ends, connected with BBS and OSA respectively, and the grating is suspended in full contact with $\mathrm{NaCl}$ solution. The grating is placed in $1 \mathrm{~L}$ of clear

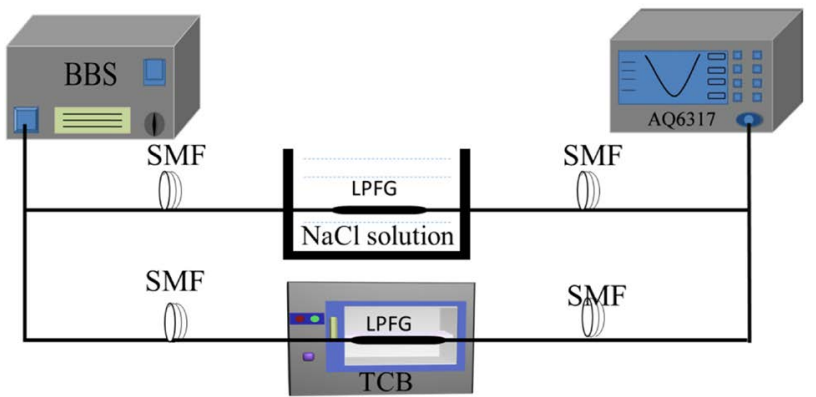

Figure 1. Measurement structure of LPFG temperature and concentration. 
water, and $\mathrm{NaCl}$ is gradually added to change the concentration of solution. As shown in Figure 2, in the range of $0 \%-17 \%$ concentration, with the increase of $\mathrm{NaCl}$ solution concentration, the central wavelength of the grating drifts to the short wavelength direction. For each change of solution concentration of $1 \%$, the central wavelength drifts to the short wave direction averagely by $0.14 \mathrm{~nm}$, which has a good linear relationship.

In the LPFG temperature measurement experiment, the temperature change of grating is realized by temperature control box. The temperature measurement range is $40^{\circ} \mathrm{C}-90^{\circ} \mathrm{C}$. As shown in Figure 3, with the increase of temperature, the coupling wavelength drifts to the long wavelength direction. The wavelength shift is $0.074 \mathrm{~nm}$ for each degree of temperature rise, and the linear fitting degree is 0.99 .

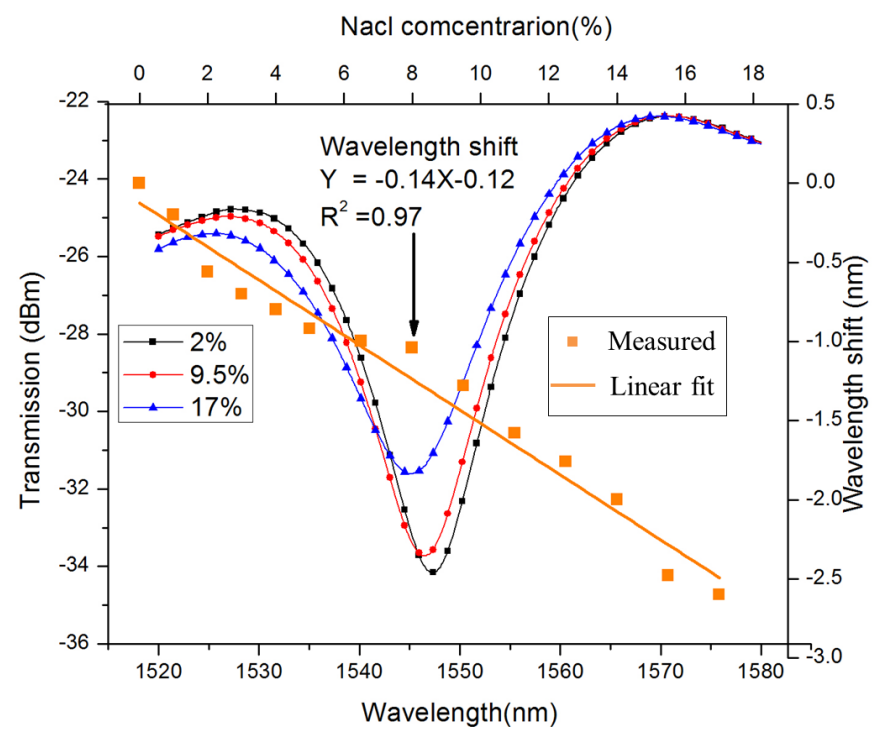

Figure 2. Measurement results of LPFG concentration.

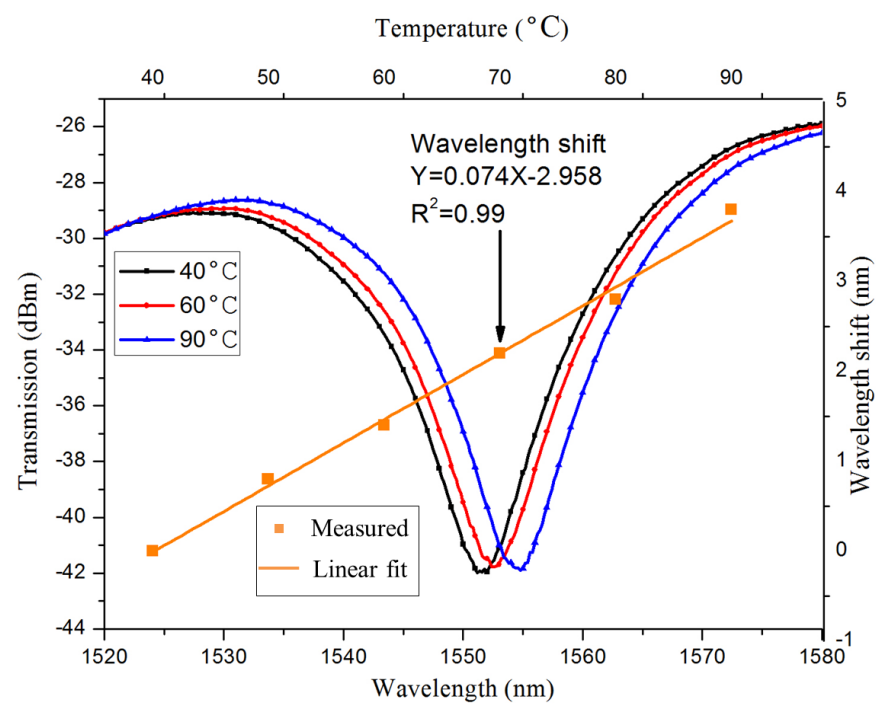

Figure 3. Measurement results of LPFG temperature. 
As shown in Figure 4, single mode and multimode fibers are selected as the intermediate connecting fibers in this experiment. By manually adjusting the fusing machine, the single mode fibers are fused into the core of multimode fibers. More fundamental mode energy in single mode fibers can stimulate the high-order modes in multimode fibers, and the redistributed energy can be fully coupled output.

The temperature measurement step is the same as that of LPFG. The measured results are shown in Figure 5. With the increase of temperature, the coupling wavelength drifts to the short wavelength direction. The wavelength shift is $0.077 \mathrm{~nm}$ for each degree of temperature rise, and the linear fitting degree is 0.96 .

The sensitivity parameters of LPFG and SMS structures are cascaded as shown in Figure 6.

The concentration coefficient $K_{R I_{L P F G}}$ of LPFG is $0.14 \mathrm{~nm} / \%$, the concentration coefficient $K_{R_{S M S}}$ of SMS is 0 , the temperature coefficient $K_{T_{L P F G}}$ of LPFG is $0.074 \mathrm{~nm} /{ }^{\circ} \mathrm{C}$, and the temperature coefficient $K_{T_{S M S}}$ of SMS is -0.077 $\mathrm{nm} /{ }^{\circ} \mathrm{C}$. Formula 6 can be obtained by substituting each sensitivity into the matrix.

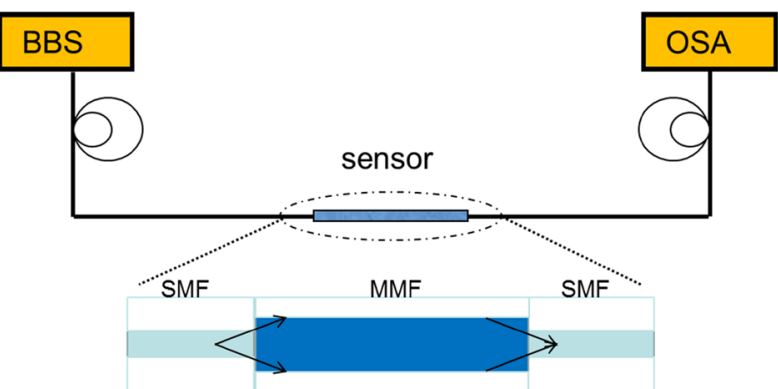

Figure 4. SMS structure.

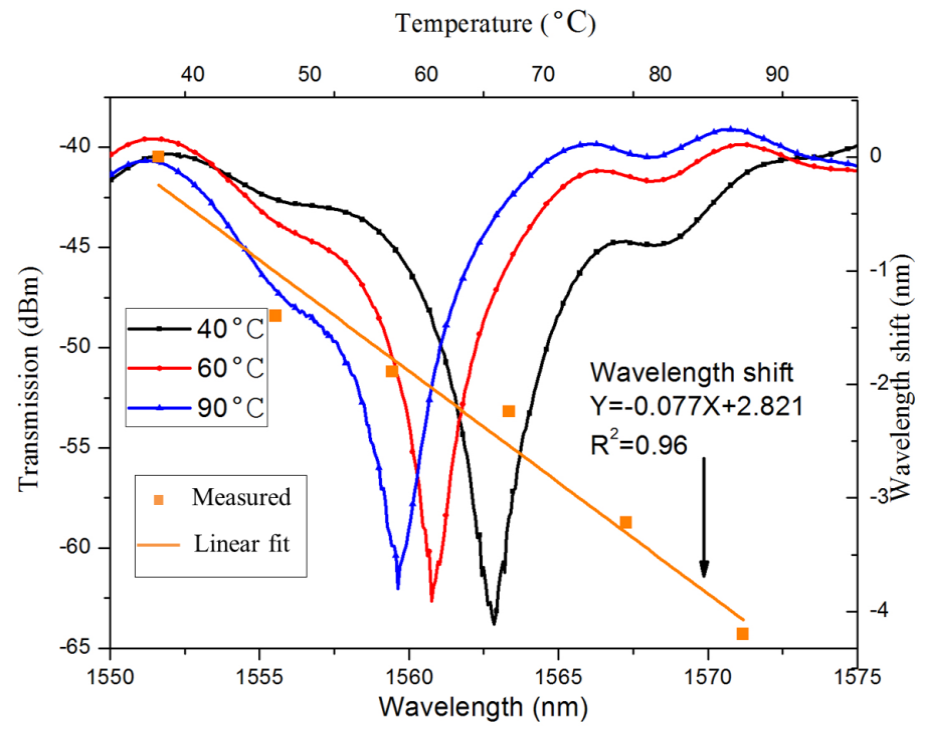

Figure 5. Measurement results of SMS temperature. 


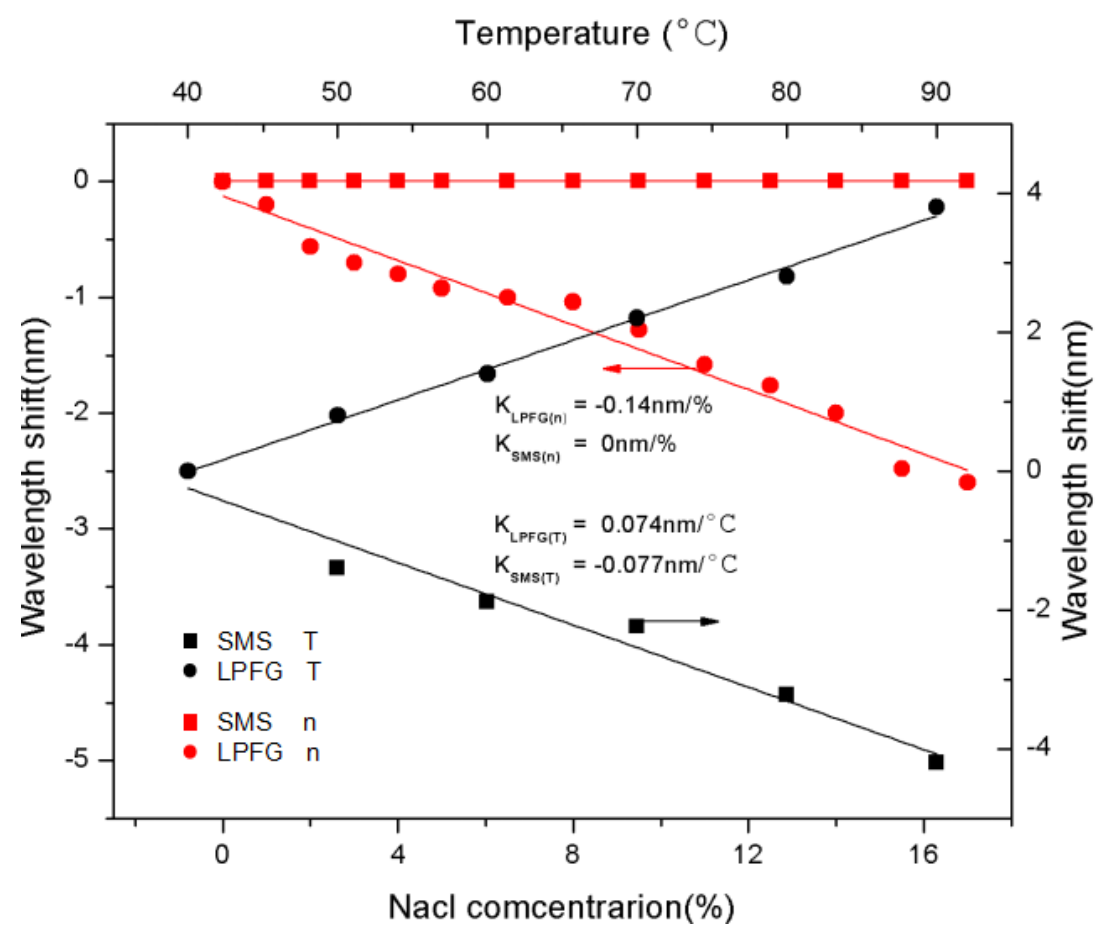

Figure 6. Cascade of sensitivity parameters for LPFG and SMS.

$$
\left[\begin{array}{c}
\Delta \lambda_{L P F G} \\
\Delta \lambda_{S M S}
\end{array}\right]=\left[\begin{array}{cc}
0.074 & 0.14 \\
-0.077 & 0
\end{array}\right]\left[\begin{array}{c}
\Delta T \\
\Delta R I
\end{array}\right]
$$

According to the change of coupling wavelength between LPFG and SMS, the change of solution concentration and temperature can be determined simultaneously when measuring solution concentration, thus eliminating the interference of temperature when measuring solution concentration.

\section{Conclusion}

In addition, a hybrid fiber structure composed of SMS structure and LPFG is proposed in this paper. The temperature-compensated solution concentration measurement is realized. The device solves the problem of temperature crosstalk when measuring solution concentration properly, and it has potential application prospects in biological and chemical domains. The center wavelength of LPFG will drift $0.14 \mathrm{~nm}$ in short wave direction with the concentration of $\mathrm{NaCl}$ solution changing by $1 \%$. The coupling wavelength of SMS structure does not change with the change of $\mathrm{NaCl}$ concentration. The temperature coefficients of LPFG and SMS structures are $0.074 \mathrm{~nm} /{ }^{\circ} \mathrm{C}$ and $-0.077 \mathrm{~nm} /{ }^{\circ} \mathrm{C}$, respectively. A dual-wavelength matrix of sensitivity is constructed to realize simultaneous measurement of solution temperature and concentration.

\section{Acknowledgements}

This work was supported by the National Natural Science Foundation of China (NSFC-61805005). 


\section{Conflicts of Interest}

The authors declare no conflicts of interest regarding the publication of this paper.

\section{References}

[1] Fiore, V., Calabrese, L., Proverbio, E., et al. (2017) Salt Spray Fog Ageing of Hybrid Composite/Metal Rivet Joints for Automotive Applications. Composites Part B, 108, 65-74. https://doi.org/10.1016/j.compositesb.2016.09.096

[2] Rajakumar, S. and Balasubramanian, V. (2018) Corrosion Performance of Friction Surfaced Nickel Aluminium Bronze (NAB) Alloy under Erosion Corrosion and Salt Fog Environment. Corrosion Engineering, Science and Technology, 53, 21-26. https://doi.org/10.1080/1478422X.2018.1425178

[3] Scalici, T., Fiore, V. and Valenza, A. (2018) Experimental Assessment of the Shield-to-Salt-Fog Properties of Basalt and Glass Fiber Reinforced Composites in Cork Core Sandwich Panels Applications. Composites Part B, 53, 29-36. https://doi.org/10.1016/j.compositesb.2018.02.021

[4] Ling, Q. and Gu, Z.T. (2019) Simultaneous Detection of SRI and Temperature with a FM-LPFG Sensor Based on Dual-Peak Resonance. Journal of the Optical Society of America B, 36, 2210-2215. https://doi.org/10.1364/JOSAB.36.002210

[5] Koyama, O., Matsui, M., Kagawa, T., et al. (2019) Low-Cost Optical Fiber Temperature-Sensing System Employing Optical Transceivers for Ethernet and Long-Period fiber Grating. Applied Optics, 58, 2366-2371. https://doi.org/10.1364/AO.58.002366

[6] Dandapat, K., Tripathi, S.M. and Chinfooroshan, Y. (2016) Compact and Cost-Effective Temperature-Insensitive Bio-Sensor Based on Long-Period Fiber Gratings for Accurate Detection of E. coli Bacteria in Water. Optics Letters, 41, 29-36. https://doi.org/10.1364/OL.41.004198

[7] Huang, J., Lan, X.W. and Kaur, A. (2014) Temperature Compensated Refractometer Based on a Cascaded SMS/LPFG. Sensors and Actuators B: Chemical, 41, 384-387. https://doi.org/10.1016/j.snb.2014.03.062

[8] Huang, J., Lan, X.W. and Kaur, A. (2016) Long Period Fiber Grating Nano-Optrode for Cancer Biomarker Detection. Biosensors and Bioelectronics, 80, 590-600. https://doi.org/10.1016/j.bios.2016.02.021

[9] Yin, G.L., Wang, Y.P., Liao, C.R., et al. (2015) Simultaneous Refractive Index and Temperature Measurement with LPFG and Liquid-Filled PCF. IEEE Photonics Technology Letters, 27, 375-378. https://doi.org/10.1109/LPT.2014.2375337

[10] Dong, X.R., Xie, Z., Song, Y.X., et al. (2017) High Temperature-Sensitivity Sensor Based on Long Period Fiber Grating Inscribed with Femtosecond Laser Transversal-Scanning Method. IEEE Photonics Technology Letters, 15, 090602-1-5. https://doi.org/10.3788/COL201715.090602

[11] Yang, B.Y., Niu, Y.X. and Yang, B.W. (2018) High Sensitivity Curvature Sensor with Intensity Demodulation Based on Single-Mode-Tapered Multimode-Single-Mode Fiber. IEEE Sensors Journal, 18, 1094-1099. https://doi.org/10.3390/s18072396

[12] Xing, R., Dong, C.B., Wang, Z.X., et al. (2018) Simultaneous Strain and Temperature Sensor Based on Polarization Maintaining Fiber and Multimode Fiber. Optics and Laser Technology, 102, 1094-1099.

https://doi.org/10.1016/j.optlastec.2017.12.013 